\title{
FOLIA
}

Amazónica

Revista del Instituto de Investigaciones

de la Amazonía Peruana

\section{DIAGNÓSTICO DE LA COMERCIALIZACIÓN INTERNACIONAL DE FAUNA SILVESTRE EN LORETO, PERÚ}

\author{
Joao D. FREITAS ${ }^{1}$, Pedro VASQUEZ ${ }^{2}$ \\ 1. Departamento de Manejo Forestal. Facultad de Ciencias Forestales. Universidad Nacional Agraria \\ La Molina, UNALM. Av. La Molina s/n, La Molina, Lima, Perú. dfreitas@lamolina.edu.pe \\ 2. Centro de Datos para la Conservación (CDC-UNALM). Departamento de Manejo Forestal. \\ Facultad de Ciencias Forestales. Universidad Nacional Agraria La Molina. Av. La Molina s/n, \\ La Molina, Lima, Perú. cdc@lamolina.edu.pe
}

\section{RESUMEN}

El comercio internacional de fauna silvestre tiene una amplia historia resultando en la casi desaparición de algunas poblaciones. Esta actividad es una importante alternativa económica, tanto para la conservación de las especies como para su aprovechamiento sostenible por las comunidades rurales. Se realizó un diagnóstico de las exportaciones entre el 2001 y 2017, evaluando la cadena de valor, especies comercializadas, modalidades de exportación, oferta y demanda, destinos, categorías de amenaza nacional e internacional de las principales especies y percepción de involucrados en fauna silvestre. Información sobre la comercialización internacional se obtuvo mediante el sistema de operatividad aduanera, la percepción mediante entrevistas y la situación actual de las especies mediante los grados de amenaza nacional e internacional. En la cadena de valor se identificaron debilidades a ser mejoradas, como evitar pérdida de datos, garantizar la trazabilidad del recurso y mejorar la articulación entre instituciones públicas y privadas permitiendo un mejor control del comercio. El valor de las exportaciones asciende a US $\$ 16,672,657.00$, cuatroítems conforman el 93.1\% de este valor, taricaya (Podocnemis unifilis) es la principal, cuyo destino es China y Hong Kong, seguido de cueros de pecarí (Tayassu pecari y Pecari tajacu) hacia Italia, iguana cabeza roja (Dracaena guianensis) a Estados Unidos y la tortuga matamata (Chelus fimbriata) a China y Hong Kong cuya demanda se 
ha incrementado en casi todos los casos. El comercio de estas especies no pone en riesgo sus poblaciones naturales y se considera una actividad importante a la economía regional y nacional.

PALABRAS CLAVE: exportación de fauna, taricaya, pecaríes, iguana cabeza roja, tortuga matamata.

\title{
DIAGNOSIS OF THE INTERNATIONAL WILDLIFE TRADE IN LORETO, PERÚ
}

\begin{abstract}
The international trade in wildlife has a long history resulting in the near disappearance of some populations. This activity is an important economic alternative, both for the conservation of the species and for their sustainable use by the rural communities. A diagnosis of exports was carried out between 2001 and 2017, evaluating the value chain, commercialized species, export modalities, supply and demand, destinations, national and international threat categories of the main species and perception of those involved in wildlife. Information on international trade was obtained through the customs operation system, the perception through interviews and the current status of the species through the national and international levels of threat. In the value chain weaknesses were identified to be improved, such as avoiding data loss, guaranteeing the traceability of the resource and improving the articulation between public and private institutions allowing better control of trade. The value of exports amounts to US $\$ 16,672,657.00$, four items make up $93.1 \%$ of this value, taricaya (Podocnemis unifilis) is the main one, whose destination is China and Hong Kong, followed by pecari hides (Tayassu pecari and Pecari tajacu) to Italy, red head iguana (Dracaena guianensis) to the United States and matamata tortoise (Chelus fimbriata) to China and Hong Kong whose demand has increased in almost all cases. Trade in these species does not put their natural populations at risk and is considered an important activity for the regional and national economy.
\end{abstract}

KEYWORDS: wildlife export, taricaya, peccaries, red head iguana, matamata tortoise. 


\section{INTRODUCCIÓN}

Loreto se encuentra en una posición privilegiada en términos de biodiversidad (Pitman et al., 2013). Ojasti (2000) reconoce que los bienes producidos por la biodiversidad satisfacen las necesidades de alimentación, salud y protección, sin embargo, la sociedad humana ha fundamentado su sistema de valoración en el valor tangible o económico de las cosas. Según lo expresado por Ojasti (2000), la fauna silvestre es la principal fuente de proteínas, y además posee valor ecológico, desde los polinizadores hasta los depredadores cumplen un rol en el ecosistema, sumado a la importancia religiosa, cultural, los valores de recreación y educación.

TRAFFIC (2018) determinó que la alta y creciente demanda de productos y sub productos de fauna silvestre se destina principalmente para alimento, mascotas, artículos de moda e investigación. Así mismo SERFOR (2017) resalta que esta demanda ocasionó que el tráfico de fauna sea una de las actividades ilícitas más lucrativas junto al tráfico de drogas y tráfico de armas afectando directamente a la biodiversidad y a la salud de los ecosistemas. Según la CITES (2018), la regulación del comercio internacional es una de las medidas que permiten fomentar el uso sostenible de los recursos naturales.

Hvidber-Hansen (1970) investigó sobre la situación del comercio de pieles de animales en Amazonía peruana, mientras se encontraba protegida por la Resolución Ministerial N 5056 70-AG, concluyendo que esta prohibición carecía de sentido y en su lugar propuso regulaciones, ya que una gran parte de la población dependía de los productos de la fauna silvestre y subproductos, principalmente del comercio de cueros de pecaríes, cocodrílidos, venados entre otras especies; cuatro años más tarde, se exceptuaron aquellas especies que eran tradicionalmente utilizadas por el poblador amazónico mediante el Decreto Supremo No $934-73-A G$.

En Loreto, las regulaciones emitidas para la conservación de recursos naturales, sumado a experiencias exitosas de manejo, han logrado que algunas especies recuperen sus poblaciones y sean comercializadas a nivel internacional bajo las regulaciones actuales y sin vulnerar las poblaciones silvestres. Bodmer et al. (1997) destacan que dos exitosas iniciativas pueden verse en la exportación de crías de taricaya (Podocnemisunifilis) y cueros de sajino y huangana (Tayassu pecari y Pecari tajacu) además afirman que el manejo de tortugas amazónicas y carne de monte poseen un alto impacto económico y social en el poblador amazónico; sin embargo, es importante conocer mayores detalles de las implicancias económicas, sociales y ambientales de la actividad.

El objetivo principal de esta investigación fue realizar un diagnóstico de la comercialización internacional de fauna silvestre entre 20012017 en Loreto periodo de vigencia de dos leyes en materia forestal y de fauna silvestre. Se logró mediante la evaluación de la cadena de valor de comercialización de fauna silvestre, determinación de las principales especies comercializadas, las modalidades de exportación, la oferta y la demanda en el período 2001-2017 y los principales destinos de exportación, una revisión del estado de conservación nacional e internacional de las principales especies comercializadas.

\section{MATERIALES Y MÉTODOS}

\section{ÁREA DE ESTUDIO}

El consideró únicamente la comercialización resultante del departamento de Loreto, ubicado en el Noreste de Perú, con una población de 1, 058, 900 habitantes (INEI, 2017). 


\section{OBTENCIÓN DE INFORMACIÓN}

Mediante las partidas arancelarias 0101, 0102, 0103 y 0104 se logró identificar 44 subpartidas, las cuales se emplearon para obtener información de las exportaciones de fauna silvestre procedente de Loreto mediante el sistema de operatividad aduanera de la SUNAT, se obtuvo un total de 2293 registros de exportaciones, sumado a la información obtenida de SERFOR asciende a un total de 3819 registros, la base de datos de comercio CITES (https://trade.cites.org/) se empleó como datos de apoyo a lo obtenido

\section{ANÁLISIS DE INFORMACIÓN}

Mediante el diagnóstico de la cadena de valor y el mapeo de actores clave, se determinó el valor de las exportaciones, cantidades exportadas y modalidad de exportación, permitiendo determinar la oferta y demanda, el país de destino, finalmente se hizo una revisión de las categorías de conservación de las principales especies exportadas, bajo las categorías de amenaza nacional (D.S N ${ }^{\circ}$ 004-2014-AG) e internacional (IUCN Red List, CITES). Adicionalmente se buscó conocer la percepción de los involucrados en la fauna silvestre mediante entrevistas.

\section{RESULTADOS}

\section{CADENA DE VALOR Y MAPA DE ACTORES EN LA COMERCIALIZACIÓN DE FAUNA SILVESTRE EN LORETO}

Tomando en consideración lo presentado por Llellish (2002) y Fang et al. (2008) se elaboró la cadena de valor con los puntos de procedencia de productos y subproductos de fauna silvestre: área natural protegida de uso directo (Reserva Nacional y Reserva Comunal, ACR), área de manejo comunal, cazadores (subsistencia) y

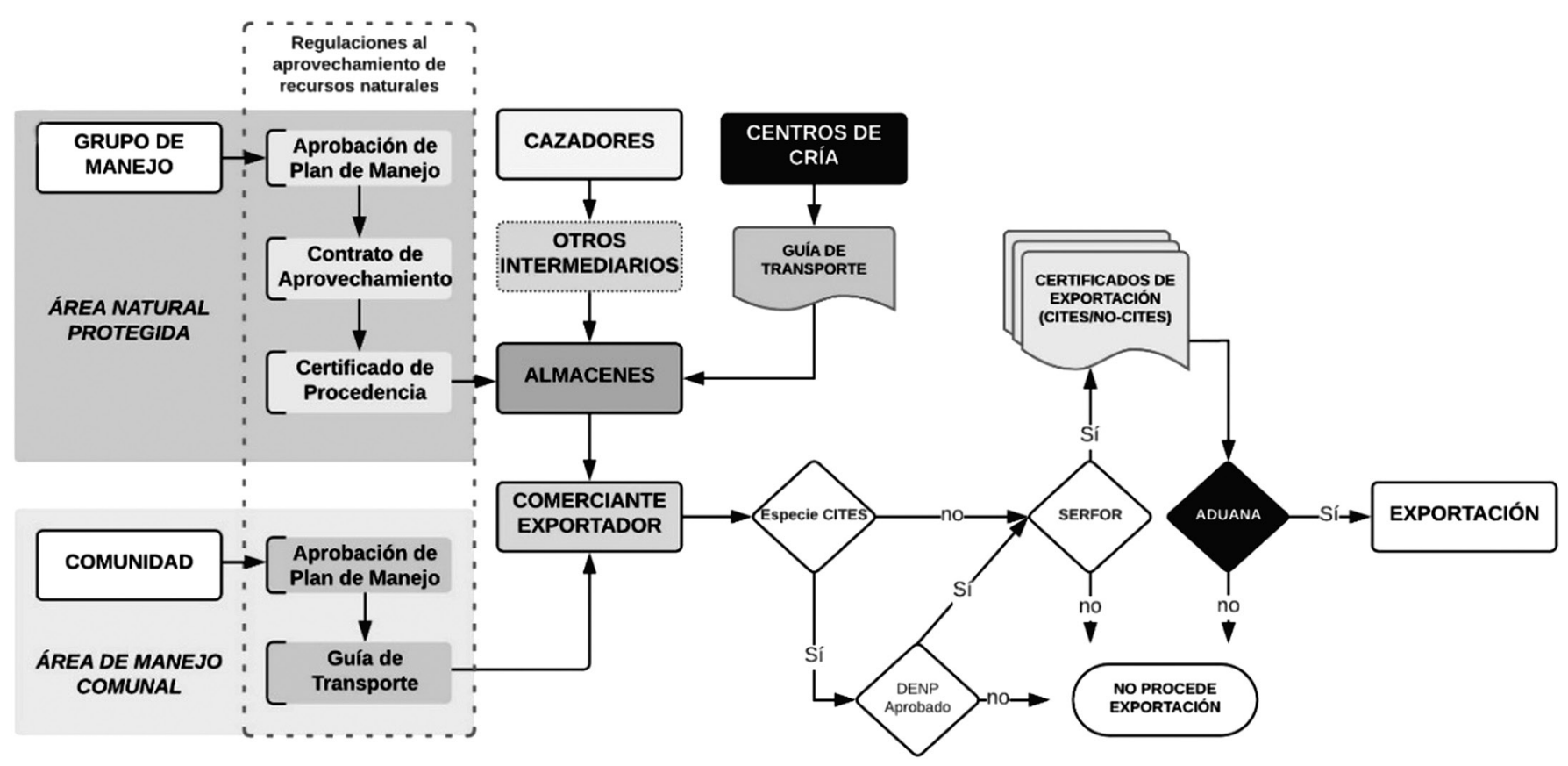

Figura 1. Esquema de comercialización de productos y sub productos de fauna silvestre. 
los centros de cría (zoocriaderos), los demás elementos que componen el mapa de actores en la cadena de valor se muestran en la Figura 1.

Las Áreas Naturales Protegidas (Reserva Nacional Pacaya Samiria y Reserva Comunal Purús) son las principales fuentes de abastecimiento de taricaya (Podocnemis unifilis). Los centros de cría aportan un menor porcentaje en comparación a las áreas naturales protegidas

\section{PRINCIPALES ESPECIES COMERCIALIZADAS}

Las exportaciones de taricaya aportan el $48.8 \%$ del valor total $(\$ 8,131,316.00)$ y cuero de pecaríes cuyas exportaciones representan el $36.8 \%$ (\$6, 143, 242. 00); mientras que, las exportaciones de tortuga matamata aporta el $3.8 \%$ del valor $(\$ 635,286.00)$ e iguana cabeza roja con $3.7 \%$ del valor $(\$ 613,858.00)$, estos recursos representan el $93.1 \%$ del valor total de las exportaciones desde Loreto en el periodo 2001 - 2017, dicho monto asciende a la suma de \$ $15,523,702.00$. Finalmente el $6.9 \%$ restante del valor total $(\$ 1,148,955.00)$, está compuesto por reptiles, psitaciformes, dendrobátidos, primates, entre otros.

\section{DESTINO DE LAS EXPORTACIONES}

Las tablas 1, 2, 3 y 4, detalla el valor de cada exportación según los destinos, el porcentaje que representa cada exportación y el valor total de las mismas, estos datos representan las exportaciones en el período 2001-2017.

\section{CATEGORÍAS DE AMENAZA DE LAS PRINCIPALES ESPECIES COMERCIALIZADAS}

\section{Podocnemis unifilis 'taricaya'}

Se encuentra en el Apéndice II de la CITES, eso implica que la especie no enfrenta el peligro de extinción, pero podría llegar, a menos que el comercio se encuentre bajo estricta reglamentación para que su utilización sea
Tabla 1. Destino y valor de las exportaciones de taricaya (2001-2017).

\begin{tabular}{|l|r|r|}
\hline Taricaya & \multicolumn{1}{|c|}{$\%$} & \multicolumn{1}{c|}{ Valor } \\
\hline Hong Kong & 70.18 & $\$ 5,706,460.00$ \\
\hline China & 27.21 & $\$ 2,212,846.00$ \\
\hline España & 0.79 & $\$ 64,608.00$ \\
\hline Japón & 0.68 & $\$ 55,625.00$ \\
\hline Taiwán & 0.59 & $\$ 48,207.00$ \\
\hline Alemania & 0.25 & $\$ 20,501.00$ \\
\hline Italia & 0.14 & $\$ 11,650.00$ \\
\hline Mexico & 0.07 & $\$ 5,535.00$ \\
\hline EEUU & 0.03 & $\$ 2,650.00$ \\
\hline Bélgica & 0.02 & $\$ 1,537.00$ \\
\hline Holanda & 0.02 & $\$ 1,250.00$ \\
\hline Malasia & 0.004 & $\$ 300.00$ \\
\hline Rep. Checa & 0.002 & $\$ 147.00$ \\
\hline Valor total & $\mathbf{1 0 0}$ & $\$ \mathbf{1 3 1 , 3 1 6 . 0 0}$ \\
\hline
\end{tabular}

Tabla 2. Destino y valor de las exportaciones de cuero de pecaríes (2001-2017).

\begin{tabular}{|l|r|r|}
\hline Cuero de pecaríes & \multicolumn{1}{|c|}{$\%$} & \multicolumn{1}{c|}{ Valor } \\
\hline Italia & 90.89 & $\$ 5,583,315.00$ \\
\hline Alemania & 6.45 & $\$ 396,378.00$ \\
\hline Hungría & 1.7 & $\$ 104,470.00$ \\
\hline EEUU & 0.62 & $\$ 38,033.00$ \\
\hline Japón & 0.33 & $\$ 20,276.00$ \\
\hline Hong Kong & 0.01 & $\$ 668.00$ \\
\hline Francia & 0.002 & $\$ 102.00$ \\
\hline Valor total & 100 & $\mathbf{\$ 6 , 1 4 3 , 2 4 2 . 0 0}$ \\
\hline
\end{tabular}

compatible con la supervivencia de la especie. La Lista Roja de la Unión Internacional para la Conservación de la Naturaleza - UICN y en el Decreto Supremo N 004-2014-AG, la ubican bajo la categoría de Vulnerable (VU), lo que significa 
Tabla 3. Destino y valor de las exportaciones de tortuga matamata (2001-2017).

\begin{tabular}{lrr}
\hline Tortuga matamata & \multicolumn{1}{l}{$\%$} & \multicolumn{1}{l}{ Valor } \\
\hline Hong Kong & 46.46 & $\$ 295,136.00$ \\
EEUU & 16.58 & $\$ 105,322.00$ \\
China & 9.42 & $\$ 59,820.00$ \\
Japón & 7.64 & $\$ 48,545.00$ \\
España & 6.18 & $\$ 39,236.00$ \\
Mexico & 4.48 & $\$ 28,480.00$ \\
Taiwan & 3.67 & $\$ 23,340.00$ \\
Malasia & 1.37 & $\$ 8,720.00$ \\
Italia & 1.18 & $\$ 7,500.00$ \\
Alemania & 0.89 & $\$ 5,632.00$ \\
Reino Unido & 0.88 & $\$ 5,600.00$ \\
Francia & 0.54 & $\$ 3,400.00$ \\
Chile & 0.4 & $\$ 2,565.00$ \\
Indonesia & 0.3 & $\$ 1,900.00$ \\
Canada & 0.01 & $\$ 90.00$ \\
\hline Valor total & 100 & $\$ 635,286.00$
\end{tabular}

que según la evidencia disponible enfrenta un alto riesgo de extinción en estado silvestre.

\section{Tayassu pecari 'Huangana' y Pecari tajacu 'Sajino'}

Según la CITES, ambas especies se encuentran en el Apéndice II, lo que significa que no enfrentan el peligro de extinción, pero podrían llegar, a menos que el comercio (de sus pieles) se encuentre bajo estricta reglamentación para que su utilización sea compatible con la supervivencia de la especie.

Lista Roja de la UICN ubica a la huangana bajo la categoría de Vulnerable (VU) lo que significa que según la evidencia disponible enfrenta un alto riesgo de extinción en estado silvestre. Además, según el DS $\mathrm{N}^{\circ}$ 004-2014-AG (2014) se ubica en la categoría de Casi Amenazado (NT) esto se debe a que, cuando se ha evaluado según los criterios y no satisface, actualmente, los criterios para categorías mayores, pero está próximo o posiblemente los satisfaga en un futuro cercano. Una especie "NT" no se considera en sentido estricto como una especie amenazada. En el caso del sajino la lista roja lo ubica en la categoría de Preocupación Menor (Lc) esto ocurre cuando habiendo sido evaluado, no cumple ninguno de los criterios que definen categorías mayores. Se incluyen en esta categoría taxones abundantes de amplia distribución. No es una categoría de amenaza. Así mismo, esta especie no se encuentra categorizada bajo el DS N 004-2014-AG (2014). Perez-Peña et al. (2017) muestra el estado poblacional de estas especies.

Tabla 4. Destino y valor de las exportaciones de iguana cabeza roja (2001-2017).

\begin{tabular}{lrr}
\hline Iguana cabeza roja & \multicolumn{1}{l}{$\%$} & \multicolumn{1}{l}{ Valor } \\
\hline EEUU & 58.48 & $\$ 358,988.00$ \\
Japón & 10.19 & $\$ 62,564.00$ \\
Hong Kong & 6.96 & $\$ 42,732.00$ \\
España & 6.63 & $\$ 40,693.00$ \\
Francia & 3.48 & $\$ 21,340.00$ \\
Bélgica & 2.45 & $\$ 15,033.00$ \\
Alemania & 2.16 & $\$ 13,280.00$ \\
Rep. Checa & 2.16 & $\$ 13,230.00$ \\
Canada & 1.88 & $\$ 11,550.00$ \\
Reino Unido & 1.47 & $\$ 8,998.00$ \\
Taiwan & 1.45 & $\$ 8,910.00$ \\
Holanda & 1.25 & $\$ 7,700.00$ \\
Rusia & 1.03 & $\$ 6,300.00$ \\
Hungría & 0.16 & $\$ 1,000.00$ \\
Malasia & 0.15 & $\$ 940.00$ \\
China & 0.1 & $\$ 600.00$ \\
\hline Valor total & 100 & $\$ 613,858.00$
\end{tabular}




\section{Chelus fimbriata 'tortuga matamata'}

No se considera como especie amenazada en el Perú, no se encuentra en la Lista Roja de la UICN, ni se encuentra incluida en los Apéndices de la CITES.

\section{Dracaena guianensis 'iguana cabeza roja'}

No se encuentra bajo ninguna categoría en la Lista Roja de la UICN, ni en el DS N $004-2014-A G$. Se encuentra en el Apéndice II de la CITES, lo que significa que no enfrenta el peligro de extinción, pero podrían llegar, a menos que el comercio se encuentre bajo estricta reglamentación para que su utilización sea compatible con la supervivencia de la especie, cabe resaltar que en el Perú no fue incluida en los apéndices de la CITES.

\section{DISCUSIÓN}

DE LACADENADEVALORYMAPADEACTORES Se identificaron todos los actores en el comercio internacional de fauna silvestre, sin embargo es importante precisar lo siguiente: Los centros de cría (zoocriaderos) cumplen un papel importante en el caso de especies como anfibios, aves de rapiña, primates o iguana cabeza roja ya que cuentan con la experiencia y el conocimiento del ciclo de vida de la especie, además de contar con un mercado estos establecimientos son constantemente supervisados por la autoridad previo al otorgamiento de las guías de transporte y los permisos de exportación, además del pago similar al derecho de aprovechamiento.

Los planes de manejo, si bien resultan un importante avance para las áreas naturales protegidas en cuánto a la aprobación de los mismos, a la vez configuran una dificultad cada vez mayor en el manejo de los recursos naturales puesto que con la legislación y reglamentación actual se tiene que elaborar -y aprobar- un plan de manejo por cada recurso, zona, cuenca y/o grupo(s) de manejo, lo que ocasiona que en una sola cuenca se cuente con varios planes de manejo del mismo recurso, documentos que al final no difieren en forma ni fondo, la única diferencia se encuentra en la ubicación o zona de manejo.

Al tratarse de varios productos y sub productos de fauna silvestre que son comercializados resulta difícil determinar el precio de compra/ venta de cada uno de ellos en los distintos puntos de la cadena de valor, como el caso de los cueros de pecaríes o taricaya. Lleellish et al. (2002) determinó el movimiento de los precios en el comercio de cueros de pecaríes en el departamento de Ucayali donde el precio fue desde S/. 8 en las comunidades y caseríos hasta S/ 24.5 pagado por los exportadores a los almacenes, mientras que finalmente estos últimos exportaban a un precio de $\$ 18$.

En la Amazonía peruana, la caza de subsistencia es la principal fuente proveedora en la industria de cueros de pecaríes, la legislación actual reconoce esta actividad exclusiva de las comunidades nativas y comunidades campesinas, en cuyo caso el fin principal es la carne como fuente de proteínas mientras que los cueros son un subproducto que viene generando un gran interés desde el siglo pasado y logrando exportar millones de cueros (Grimwood 1969, Córdova 1958) de manera ininterrumpida hasta el año 1973 en que se estableció la restricción a la caza con fines comerciales, mediante el Decreto Supremo $\mathrm{N}^{\circ}$ 934-73-AG (1973), que dispuso por tiempo indefinido la caza y/o captura de todas las especies de animales silvestres de la Región de la Selva, y prohibió la comercialización de los animales, productos y despojos de los mismos, dentro y fuera del país.

De acuerdo al reglamento de la Ley $\mathrm{N}^{\circ} 29763$ (2011), la acreditación del origen legal de los productos y subproductos de fauna silvestre mediante guías de transporte, autorizaciones, guía de remisión o documentos de importación o 
reexportación constituyen una buena medida que permite asegurar la trazabilidad de los productos desde su origen y en cada una de las etapas de la cadena productiva; sin embargo, durante esta investigación se han identificado algunos problemas durante la recopilación y manejo de la información.

El contar con información actualizada resulta importante para una adecuada interpretación de la situación actual y toma de decisiones. Sin embargo, al tratarse de información que se genera en distintos puntos de un territorio y al no contar con un sistema 'en línea' que permita alimentar constantemente una base de datos, termina generando dificultades en distintas fases del proceso. Todo ello sumado a las dificultades propias de cada departamento (ATFFS o GORE's), como la falta de personal debidamente capacitado en materia de manejo de recursos naturales, que además conozca el manejo administrativo y sobre comercio de especies CITES, o la falta de equipamiento necesario y un sistema interno del manejo de la información.

\section{DE LAS PRINCIPALES ESPECIES COMERCIALIZADAS}

Todas las descripciones comerciales consignadas fueron exportadas como especímenes vivos - excepto los cueros de pecaríes - en cajas de distinto material (plástico, madera, tecnopor) que varió de acuerdo al espécimen, cantidad y exportador, y la única vía de transporte fue la aérea, como se sabe la ciudad de Iquitos no cuenta con conexión por carreteras por lo tanto la exportación de especímenes vivos se realiza por vía aérea hacia los principales destinos con conexión en Lima. Si bien es cierto los cueros de pecaríes no fueron exportados desde Iquitos, se considera este recurso porque Loreto es uno de las regiones de origen del recurso hacia la ciudad de Lima en estado seco salado, ya que Loreto no cuenta con la experiencia ni la tecnología para procesar los cueros, estos son transportados en estado seco salado hacia la Lima o Arequipa para luego ser exportados. En este caso se incluye el valor de las exportaciones de pecaríes, mas no se incluyen las cantidades, ya que estos fueron exportados bajo diferentes unidades (unidad, kilos, $\mathrm{m}^{2}$ ) por lo que no fue posible unificar estos valores se tomó como referencia el valor de las exportaciones (US\$).

\section{DEL DESTINO DE LAS EXPORTACIONES}

El comercio internacional de taricaya, tiene como principal destino Hong Kong con $70.18 \%$ del valor total, lo que representa US\$5, 706,460. Seguido de China con 27.21\% del valor total y US\$2, 212,846. Esta diferencia entre China y Hong Kong se debe a que Hong Kong tiene una economía capitalista y un sistema político democrático, con un gran nivel de autonomía, a pesar de eso gran parte de sus decisiones están supeditadas a la aprobación del gobierno de China, con ideología comunista. Esto producto de un tratado entre China y la corona británica en el siglo XIX conocido como Tratado de Nankín, desde 1997 Hong Kong se convierte en parte de un país comunista (China) con economía capitalista y política democrática.

Según la Organización de las Naciones Unidas para la Alimentación-FAO, durante el II Congreso Mundial del Cuero que tuvo lugar en Italia en 2016, la cadena de valor de la industria del cuero significa un movimiento económico de 80000 millones de dólares, muy por encima de la cadena de valor del caucho y del algodón (10 000 millones de dólares). Según la base de datos sobre comercio de las Naciones Unidas (UN Comtrade Database), en el año 2017, Italia tuvo importaciones por un total de 3,500 millones de dólares en productos de cuero, los cuales se exportaron como productos terminados por un valor de 9,200 millones de dólares. En la tabla 2 se detalla el destino de las exportaciones de cuero de pecaríes, teniendo como principal destino 
Italia (90.89\%) con un valor de US\$ 5, 583,315 del valor total de las exportaciones.

Al igual que ocurre con las exportaciones de Taricaya, Hong Kong es el principal mercado de destino de las exportaciones de tortuga matamata, el $46.46 \%$ del valor de las exportaciones (US\$ 295,136.00).

La iguana cabeza roja es tiene como principal destino a EEUU y China, aunque el mayor valor de las exportaciones corresponde al país norteamericano (46.46\%) con US\$295,136.00, si bien es cierto estas cifras son mucho menores en comparación a otras especies ya que representa el 3.7\% del valor total de las exportaciones de fauna silvestre, puede considerarse como una especie con potencial para el comercio.

Se sabe que el comercio de fauna silvestre y subproductos es una práctica recurrente en el mundo, tanto de manera ilegal (tráfico), la cual se desarrolla de manera amplia en la estrategia para reducir el tráfico de fauna silvestre en el Perú (SERFOR 2017), como de manera legal. En el período 1950-1956 en la selva peruana se desarrollaba una importante industria extractiva con los cueros y pieles de la fauna silvestre y se recopiló datos referente a la exportación de pieles y cueros de mayor demanda internacional, las especies fueron las siguientes: huangana (Tayassu pecari), sajino (Pecari tajacu), ronsoco (Hydrochaeris hydrochaeris), venado (Mazama americana), jaguar (Panthera onca), tigrillo (Leopardus wiedii), nutria (Lontra longicaudis) y lobo de río (Pteronura brasiliensis), las exportaciones alcanzaron la cifra de 1100246 pieles y cueros entre 1ra y 3ra calidad (Cordova 1958), no se tiene información del destino de estas exportaciones.

En 1969, las exportaciones de pieles y cueros alcanzó la cifra de 308743 cuyo principal destino fue Alemania (198024) y EEUU (84 713), las principales especies objeto de estas exportaciones fueron sajino (155 100), huangana
(68 400) y venado (27 083), además en aquella época se comercializaba pieles de especies que pasaron a estar protegidas, prohibiendo todo tipo de comercio, tal es el caso de lobo de río, cuatro especies de felinos (Panthera onca, Leopardus wiedii, Leopardus pardalis, y Puma concolor), nutria de río ronsoco y caimán negro (Melanosuchus niger), en este caso la calidad de las pieles no fue una variable de los datos que se recopilaron (Hvidberg-Hánsen, 1970). En 1990 se reportó la producción (no exportación) de 36 504 cueros de sajino, huangana y venado, siendo el sajino la especie que más aportó (22 098), de la misma manera no se tiene información de los destinos de estas exportaciones ni la calidad de los cueros (MINAGRI 1991).

\section{DEL ESTADO DE CONSERVACIÓN DE LAS PRINCIPALES ESPECIES}

\section{Pododnemis unifilis 'taricaya'}

Actualmente la especie se viene manejando con éxito en dos áreas naturales protegidas en Loreto desde los años 90 (Soini 1999), sumado a los esfuerzos realizados en los centros de cría, no se puede asegurar que la especie se ha recuperado porque se requiere estudios más detallados para tal afirmación. La especie se viene manejando en los sectores de: cuenca Yanayacu-Pucate, cocha Jacinto y el caño Yanayaquillo, cuenca del Pacaya, cuenca Yayanacu Grande, cocha Pinchi, caño Chiric y quebrada Yanayacu Grande, cuenca Samiria, cocha Cuyo Coyote, en la Reserva Nacional Pacaya Samiria (RNPS) y en los sectores de Alto Curunja y Alto Purús de la Reserva Comunal Purús (RCP).

\section{Pecari tajacu 'sajino' y Tayassu pecari 'huangana'}

Perez-Peña et al. (2017) evaluaron 33 localidades (Loreto y Ucayali) mediante transectos de banda, recorriendo un total de 14,220.5 km. P. tajacu mostró una densidad poblacional de 1.24 ind/ 
$\mathrm{km}^{2}$ y fue registrada en $96.9 \%$ de las localidades, mientras que para T. pecari la densidad fue de 1.59 ind $/ \mathrm{km}^{2}$ y se registró en $48.5 \%$ de las localidades estudiadas; además, dicho estudio incluyó la percepción de los cazadores mediante 162 entrevistas realizadas en 11 comunidades sobre la abundancia de ambas especies, reconociendo al $P$. tajacu como una especie 'abundante' y a $T$. pecari como una especie 'frecuente o abundante'.

El 21 de marzo de 2018, mediante Resolución de Dirección Ejecutiva $N^{\circ}$ 054-2018-MINAGRISERFOR/DE se aprobó la cuota máxima de comercialización de cueros de pecaríes provenientes de la caza de subsistencia por el período de un año calendario, detallando que para Loreto las cuotas son las siguientes: 25,379 cueros de sajino y 9,043 de huangana, además del pago por derecho de aprovechamiento: S/ 4.92 por cuero de sajino y $\mathrm{S} / 5.72$ por cuero de huangana.

Por otro lado, no se cuenta con información sobre el estado de las poblaciones naturales de tortuga matamata e iguana cabeza roja, tal como se menciona en los resultados, las exportaciones de estas especies proceden únicamente de zoocriaderos.

\section{CONCLUSIONES}

El comercio internacional de fauna silvestre en Loreto se encuentra con diversas fallas a lo largo de la cadena de valor, desde el origen de la información, la falta de coordinación entre instituciones públicas y dentro de los distintos niveles en las propias instituciones, además de falta de coordinación entre actores del sector público y privado.

Se identificaron fallas en el sistema actual de registro y control de permisos de exportación CITES. No existe información acerca del estado de las operaciones de los centros de cría en Loreto. La cadena de valor actual en el comercio de cueros de pecaríes ocasiona pérdidas en el proceso, además de la falta de información actualizada de los precios de venta en cada etapa.

La modalidad de exportación de todos los especímenes (excepto cuero de pecaríes) fueron vivos, por vía aérea, en cajas de madera, plástico y tecnopor, con fines de comercio (mascotas), investigación científica y exhibición. La especie con mayor valor de exportaciones es Podocnemis unifilis 'Taricaya', seguido por cueros de pecaríes (Tayassu pecari y Pecari tajacu), Chelus fimbriata 'Tortuga matamata' y Dracaena guianensis 'Iguana cabeza roja'. Las especies que componen el mayor valor de las exportaciones se encuentran en buen estado de conservación pese a los niveles históricos y actuales de comercio.

\section{BIBLIOGRAFIA CITADA}

Bodmer, R.E., Penn J., Puertas P., Moya, L. y Fang, T. 1997. Linking conservation and local people through sustainable use of natural resources: community-based management in the Peruvian Amazon. En: Harvesting wild species. F. Curtis (ed). The John Hopkins University Press. Baltimore y London. Pp. 315-358.

CITES 2018. ¿Cómo funciona la CITES? (https:// www.cites.org/esp/disc/how.php) Acceso: 19/03/2018

Cordova, D. 1958. La explotación de Cueros y Pieles de Animales Silvestres: Una importante industria en el Perú. Revista Pesca y Caza ${ }^{\circ}$ 8. Ministerio de Agricultura. Dirección de Pesquería y Caza. Lima-Perú. 94 pp.

Fang, T., Bodmer, R.E., Puertas, P.E., Mayor, P., Perez, P., Acero, R., Hayman, D.T. 2008. Certificación de pieles de pecaríes en la Amazonía Peruana. Una estrategia para la conservación y el manejo de fauna silvestre en la Amazonía Peruana. WCSDICE-DARWIN-INRENA-FUNDAMAZONÍA. 205 pp. 
Grimwood, I. 1969. Notes on the distribution and status of some Peruvian mammals. Special publication $\mathrm{N}^{\circ}$ 21. American committee for international wildlife protection and New York Zoological Society. Bronx, NY.

Hvidberg-Hansen, H. 1970. Recomendaciones sobre aspectos específicos de legislación en vida silvestre y control de comercio de pieles. Informe para la Dirección General Forestal de Caza y Tierras. Proyecto de Investigación y Capacitación Forestal en Perú $N^{\circ} 116$ del Fondo Especial para las Naciones Unidas y FAO. Lima. 21 pp.

Lleellish, M. 2002. El circuito de comercialización y los procesos de valor agregado de pieles de pecaríes. Documento INRENA, Lima. 30p

Ojasti, J. 2000. Manejo de Fauna Silvestre Neotropical. F. Dallmeier (editor). SI/MAB. Series \# 5. Smithsonian Institution/MAB Biodiversity Program, Washington D.C. 304p.

Ministerio de Agricultura (MINAGRI). 1991. Perú Forestal en Números. Lima, Perú. 161p.

Perez-Peña, P.; Riveros, M.; Mayor, P.; Ramos, M.; Aquino, R.; Lopez, L; Bodmer, R.; Bowler, M.; Antúnez, M.; Puertas, P.; Flores, G.; Garcia, G.;
Tapia, C.; Charpentier, E.; Bardales, C.; Torres, L.; Ramos, V.; Amilcar, O.; Gonzales, C.; Diaz, M.; Segura, J.; Calle, A.; Ruck, L.; Beraun, Y.; Mejia, K. 2017. Estado poblacional de sajino (Pecari tajacu) y huangana (Tayassu pecari) en la Amazonía peruana. Folia Amazónica, 26 (2) 2017:103-120.

Pitman, N., Gagliardi Urrutia, G., Clinton, J. 2013. La Biodiversidad de Loreto, Perú: El conocimiento actual de la diversidad de plantas y vertebrados terrestres. Abril 2013 Center for International Environmental Law (CIEL). 40 pp.

Servicio Nacional Forestal y de Fauna Silvestre (SERFOR) Perú 2017. Estrategia nacional para reducir el tráfico ilegal de fauna silvestre en el Perú 2017-2027: Y su plan de acción 20172022. Primera edición. Perú. 72 pp.

Soini, P. 1999. Un manual para el Manejo de Quelonios Acuáticos en la Amazonía Peruana (Charapa, taricaya, cupiso). Instituto de Investigaciones de la Amazonía Peruana. 83p TRAFFIC-Wildlife Trade. 2018. (http://www. traffic.org/trade/) Acceso: 19/03/2018

Recibido: 10 de octubre de 2018 Aceptado para publicación: 5 de diciembre de 2018

Esta obra está bajo una Licencia Creative Commons Atribución-NoComercial-SinDerivar 4.0 Internacional. 